\title{
A mixed-methods needs assessment of traumatic brain injury care in a low- and middle-income country setting: building neurocritical care capacity at two major hospitals in Cambodia
}

\author{
Ariana S. Barkley, MD, ${ }^{1}$ Laura J. Spece, MD, MS, ${ }^{2,3}$ Lia M. Barros, DNP, AGAC-NP, RN, ${ }^{2}$ \\ Robert H. Bonow, MD, ${ }^{1}$ Ali Ravanpay, MD, PhD, ${ }^{1,4}$ Richard Ellenbogen, MD, ${ }^{1}$ Phearum Huoy, MD, ${ }^{5}$ \\ Try Thy, MD, ${ }^{6}$ Seang Sothea, ISAR, MPA, ${ }^{6}$ Sopheak Pak, BSN, RN, James LoGerfo, MD, MPH, ${ }^{8,9}$ \\ and Abhijit V. Lele, MBBS, MD, MS ${ }^{10}$
}

\begin{abstract}
Departments of ${ }^{1}$ Neurological Surgery and ${ }^{2}$ Medicine, University of Washington; Departments of ${ }^{3}$ Health Services Research and Development and ${ }^{4}$ Neurological Surgery, VA Puget Sound Health Care System, Seattle, Washington; ${ }^{5}$ Department of Neurological Surgery, Khmer Soviet Friendship Hospital; ${ }^{6}$ Department of Neurological Surgery and ${ }^{7}$ Technical Office, University of Health Sciences, Phnom Penh, Cambodia; and ${ }^{8}$ Department of Medicine, Division of General Internal Medicine, and Departments of ${ }^{9} \mathrm{Global}$ Health and ${ }^{10} \mathrm{Anesthesiology}$ and Pain Medicine, University of Washington, Seattle, Washington
\end{abstract}

\begin{abstract}
OBJECTIVE The high global burden of traumatic brain injury (TBI) disproportionately affects low- and middle-income countries (LMICs). These settings also have the greatest disparity in the availability of surgical care in general and neurosurgical care in particular. Recent focus has been placed on alleviating this surgical disparity. However, most capacity assessments are purely quantitative, and few focus on concomitantly assessing the complex healthcare system needs required to care for these patients. The objective of the present study was to use both quantitative and qualitative assessment data to establish a comprehensive approach to inform capacity-development initiatives for TBI care at two hospitals in an LMIC, Cambodia.
\end{abstract}

METHODS This mixed-methods study used 3 quantitative assessment tools: the World Health Organization Personnel, Infrastructure, Procedures, Equipment, Supplies (WHO PIPES) checklist, the neurosurgery-specific PIPES (NeuroPIPES) checklist, and the Neurocritical Care (NCC) checklist at two hospitals in Phnom Penh, Cambodia. Descriptive statistics were obtained for quantitative results. Qualitative semistructured interviews of physicians, nurses, and healthcare administrators were conducted by a single interviewer. Responses were analyzed using a thematic content analysis approach and coded to allow categorization under the PIPES framework.

RESULTS Of 35 healthcare providers approached, 29 (82.9\%) participated in the surveys, including 19 physicians $(65.5 \%)$ and 10 nurses (34.5\%). The majority had fewer than 5 years of experience $(51.7 \%)$, were male $(n=26,89.7 \%)$, and were younger than 40 years of age $(n=25,86.2 \%)$. For both hospitals, WHO PIPES scores were lowest in the equipment category. However, using the NCC checklist, both hospitals scored higher in equipment $(81.2 \%$ and $62.7 \%)$ and infrastructure (78.6\% and 69.6\%; hospital 1 and 2, respectively) categories and lowest in the training/continuing education category ( $41.7 \%$ and $33.3 \%$, hospital 1 and 2 , respectively). Using the PIPES framework, analysis of the qualitative data obtained from interviews revealed a need for continuing educational initiatives for staff, increased surgical and critical care supplies and equipment, and infrastructure development. The analysis further elucidated barriers to care, such as challenges with time availability for experienced providers to educate incoming healthcare professionals, issues surrounding prehospital care, maintenance of donated supplies, and patient poverty.

CONCLUSIONS This mixed-methods study identified areas in supplies, equipment, and educational/training initiatives

\footnotetext{
ABBREVIATIONS HIC = high-income country; ICU = intensive care unit; LMIC = low- and middle-income country; NCC = Neurocritical Care; NCCU = neurocritical care unit; NCS = Neurocritical Care Society; NeuroPIPES = neurosurgery-specific PIPES; PIPES = Personnel, Infrastructure, Procedures, Equipment, Supplies; TBI = traumatic brain injury; WHO PIPES = World Health Organization PIPES.
}

SUBMITTED August 14, 2019. ACCEPTED October 11, 2019.

INCLUDE WHEN CITING Published online December 20, 2019; DOI: 10.3171/2019.10.JNS192118. 


\begin{abstract}
as areas for capacity development for TBI care in an LMIC such as Cambodia. This first application of the NCC checklist in an LMIC setting demonstrated limitations in its use in this setting. Concomitant qualitative assessments provided insight into barriers otherwise undetected in quantitative assessments.
\end{abstract}

https://thejns.org/doi/abs/10.3171/2019.10.JNS192118

KEYWORDS traumatic brain injury; neurocritical care; low-income countries; middle-income countries; needs assessment; mixed methods; capacity development; trauma

$\mathrm{E}$ VERY year an estimated 22.6 million patients suffer some form of neurological disease worldwide that warrants the expertise of a neurosurgeon. Patients who sustain a traumatic brain injury (TBI) specifically require a significant proportion of essential neurosurgical care. $^{5,12}$ Of these 22.6 million patients, an estimated 13.8 million require surgery, ${ }^{5}$ but the availability of surgical care globally is inadequate. According to the Lancet Commission on Global Surgery, as many as 5 billion people lack access to safe, affordable surgical care and anesthesia. ${ }^{9}$

Road traffic accidents with associated TBI are increasingly a major cause of death and disability worldwide, ${ }^{11}$ and low- and middle-income countries (LMICs) are disproportionately affected, with Western Pacific and Southeast Asian regions experiencing the greatest overall burden of disease..$^{911,14,20}$ Cambodia is a nation in Southeast Asia, home to 16.01 million people, and considered an LMIC based on a gross national income per capita of $\$ 1230$ US as of $2017 .{ }^{19}$ The average life expectancy is 69.4 years. ${ }^{21}$ A study conducted between 2013 and 2016 at Preah Kossamak Hospital in Phnom Penh, Cambodia, reported an increase over time in the number of patients with TBI, primarily due to road traffic accidents, ${ }^{14}$ road traffic accidents are ranked in the top 5 leading causes of death in the country. ${ }^{21}$

Optimal care for TBI requires complex processes across a healthcare system, including surgery, anesthesia, and critical care. ${ }^{1,4}$ Studies of TBI outcomes in highincome countries (HICs) have suggested a $26 \%$ increase in the mortality rate for trauma patients treated in general intensive care units (ICUs) versus ICUs with neurospecialization. ${ }^{13}$ The Neurocritical Care Society (NCS) has recently established standards for neurocritical care units (NCCUs). ${ }^{10}$ However, these standards are based on $\mathrm{HIC}$ resources. LMIC health systems experience unique barriers to fulfilling these requirements based on their neurosurgical and neurocritical care capacity. ${ }^{17}$ Evaluation of critical care capacity is not reflected in current instruments developed to assess surgical capacity, such as the World Health Organization Personnel, Infrastructure, Procedures, Equipment, Supplies (WHO PIPES) instrument, ${ }^{6}$ its neurosurgery-specific counterpart, NeuroPIPES,${ }^{16}$ and the Neurocritical Care (NCC) checklist recently published by $\mathrm{NCS}^{10}$

To inform capacity-development initiatives for TBI in an LMIC setting, we conducted a mixed-methods study to assess capacity for neurosurgical care in general, and perioperative/nonoperative TBI care in particular, at two national hospitals in Phnom Penh, Cambodia. The mixedmethods study design included administering quantitative survey instruments, together with qualitative interviews conducted with healthcare personnel at both hospitals. This assessment is the first application in an LMIC setting of the NCC checklist, an assessment tool that incorporates nonoperative and perioperative requirements for caring for the TBI population. Using this mixed-methods design, our study provides an assessment of neurosurgical TBI care in these hospitals and creates the opportunity for native healthcare providers to articulate perceived needs as they articulate their visions for improvement that would otherwise not be given voice to through purely quantitative assessments.

\section{Methods \\ Study Setting and Participants}

The two hospitals in Cambodia were selected for their features as large public tertiary referral centers with an over 1400-bed capacity between the two institutions. Both facilities provide subspecialty care, including neurosurgery, with a total of 10 practicing neurosurgeons across the two sites. Hospital 1 has a dedicated neuro-ICU, and hospital 2 has a general surgical ICU providing care for neurosurgical patients. Providers estimate that more than 1500 operative procedures are performed per year between the two institutions and that there is an emergency department census averaging approximately 200 patients seen per day, with $15 \%-20 \%$ of these cases involving head injury.

Local Cambodian coinvestigators at both hospitals assisted in the selection of study participants. Study participants included healthcare administrators, physicians, and nurses working in the emergency, neurosurgery, neurology, neurological intensive care, and medical intensive care departments at hospital 1 and the emergency, neurosurgery, neurology, and surgical intensive care departments at hospital 2.

This study was determined to be exempt from human subjects review by the University of Washington Human Subjects Division, and approval by the Cambodia National Ethics Committee for Health Research was not required. Two hospitals in Phnom Penh participated in this study. Approval from the director of each hospital was obtained to conduct interviews with employees. At both hospitals, leadership supported completion of this project as a quality improvement initiative rather than human subjects research. Consent to participate in the survey and interview was obtained from all participants.

\section{Survey and Interview Data Collection}

We utilized a mixed-methods study design incorpo- 
rating quantitative survey questionnaires and qualitative semistructured physician and nurse interviews. Three survey instruments were used: 1) the WHO PIPES, ${ }^{6}$ 2) the NeuroPIPES ${ }^{16}$ and 3) the NCC checklists. ${ }^{10}$ The WHO PIPES and NeuroPIPES checklists have 105 and 126 components, respectively, divided into categories of personnel, infrastructure, procedures, equipment, and supplies. The WHO PIPES checklist is embedded in the NeuroPIPES checklist. The NeuroPIPES checklist includes additional questions about a dedicated neurosurgery operating room, performance of various neurosurgery-specific basic procedures and neurosurgery-specific equipment such as cranial drills. An index score for both tools is calculated by tabulating the total number of components present, dividing by the number of items in the tool and multiplying by 10. The NCC checklist consists of 261 items divided into 5 main subdivisions to direct the establishment of Level 1, 2 , and 3 neurocritical care units (NCCUs). ${ }^{10}$ The main divisions consist of the following: infrastructure, personnel, equipment, processes, and training/continuing education/ research, with specifications for time limits on the availability of personnel, resources, laboratory results, and education requirements. The total number of items present were tabulated and compared to the number of total items in the checklist. A comparison of the 3 checklists is shown in Supplementary Table 1.

One interviewer administered the 3 assessment tools to all 29 study participants in a private meeting room during site visits to both hospitals. Surveys were administered in English, and answers were recorded in Microsoft Excel by the interviewer at the time of survey administration. Survey respondents were given the opportunity to provide clarification and expand on their responses, if desired. Expanded responses were recorded as qualitative responses and were analyzed together with the qualitative interview data. Administration of surveys and interviews continued until saturation was reached, as demonstrated by repetition of similar themes and comments from multiple participants across departments.

Qualitative interviews were conducted with all study participants in conjunction with or shortly after administration of the quantitative surveys. Interviews were conducted in English, and data collection by the interviewer was performed using extensive note-taking by hand and in Microsoft Excel. An open-ended interview guide structured around the PIPES subdivisions (personnel, infrastructure, procedures, equipment, and supplies) was used for this project.

\section{Statistical Analysis}

For the quantitative analysis, survey data points were tabulated for WHO PIPES and NeuroPIPES, with indices calculated by dividing the total number of items reported by the total number of items on the checklist and multiplying by 10 . The total number of items present in the NCC survey was reported as a percentage of the total number of items in each category.

The qualitative interview data were analyzed using theoretical thematic analysis following the steps described by Braun and Clarke. ${ }^{2,3}$ Open coding was used to evaluate the qualitative data collected through interview notes.
TABLE 1. Characteristics of interviewees

\begin{tabular}{lccr}
\hline Characteristic & Hospital $1^{*}$ & Hospital $2 \dagger$ & Total $(\%)$ \\
\hline Age $(\mathrm{yrs})$ & \multicolumn{3}{c}{} \\
\hline$<30$ & 9 & 2 & $11(37.9)$ \\
\hline $30-40$ & 6 & 8 & $14(48.3)$ \\
\hline$>40$ & 3 & 1 & $4(13.8)$ \\
\hline Experience (yrs) & & & \\
\hline$<5$ & 9 & 6 & $15(51.7)$ \\
\hline $5-10$ & 7 & 3 & $10(34.5)$ \\
\hline$>10$ & 2 & 2 & $4(13.8)$ \\
\hline Sex & & & \\
\hline Male & 16 & 10 & $26(89.7)$ \\
\hline Female & 2 & 1 & $3(10.3)$ \\
\hline Degree & & & \\
\hline MD or equivalent & 12 & 7 & $19(65.5)$ \\
\hline RN or equivalent & 6 & 4 & $10(34.5)$ \\
\hline
\end{tabular}

* Hospital 1 subspecialties: emergency (3 physicians; group interview), neurology (1 physician; individual interview), neurosurgery (2 residents, 2 physicians; individual interviews), neurological ICU (1 physician, 2 nurses; individual interviews), medical ICU (2 physicians, 2 nurses; both individual and a group interview), and administrative healthcare providers (1 physician, 2 nurses). $\dagger$ Hospital 2 subspecialties: emergency (2 physicians; group interview), neurology (1 physician; individual interview), neurosurgery (2 physicians, 1 nurse; individual interviews), and surgical ICU (2 physicians, 3 nurses; individual interviews).

Coding was conducted using a descriptive method to summarize the primary communicated topic for each answer. ${ }^{8}$ These coded themes were subsequently organized into the PIPES framework to facilitate reporting.

\section{Results}

\section{Characteristics of Interviewees}

Characteristics of study interviewees are shown in Table 1. At hospital 1, a total of 20 healthcare professionals were approached from the emergency department, neurology department, neurosurgery department, neurological ICU, and medical ICU, and 18 agreed to be interviewed. At hospital 2, 15 healthcare professionals were approached from the emergency department, neurology department, neurosurgery department, and surgical ICU, and 11 agreed to be interviewed. The majority of interviewees were $\leq 40$ years of age $(n=25,86.2 \%)$, male $(n=$ $26,89.7 \%)$, and physicians $(\mathrm{n}=19,65.5 \%)$ with $<5$ years of experience in their chosen fields $(n=15,51.7 \%)$.

\section{Quantitative Assessment Results}

The results of the NeuroPIPES survey are shown in Table 2, and those of the WHO PIPES checklist, which is nested within the NeuroPIPES tool, are summarized in Table 3. According to the personnel and infrastructure scores on the NeuroPIPES survey, both hospitals had the required subspecialty healthcare providers and the infrastructure to care for operative and nonoperative patients with TBI (Table 2). Hospital 1 reported access to all supply items listed, but the total index was decreased due to the lack of specified equipment and the capacity to provide 
TABLE 2. Summary of scores on the NeuroPIPES assessment survey

\begin{tabular}{lccc}
\hline \multicolumn{1}{c}{ Category } & Hospital 1 & Hospital 2 & Total Possible Score \\
\hline Personnel & 44 & 32 & NA \\
\hline Infrastructure* & 24 & 23 & 15 \\
\hline Procedure & 41 & 46 & 49 \\
\hline Supplies & 25 & 17 & 25 \\
\hline Equipment & 29 & 20 & 32 \\
\hline Total & 163 & 138 & NA \\
\hline Index $†$ & 12.9 & 10.9 & NA \\
\hline
\end{tabular}

NA = not applicable.

* There are 15 total infrastructure items; however, the number of operating rooms is added to this score, which is why the hospital scores exceed the total possible score.

$\dagger$ Calculated by taking the total score, dividing by the number of survey items $(n=126)$, and multiplying by 10 : (total $/ 126) \times 10$.

general pediatric procedures. Hospital 2 offered general procedures in the pediatric population, thus raising the procedure score; however, it reported a lack of supply and equipment items, decreasing its index score. The separate WHO PIPES assessment is shown in Table 3.

As shown in Fig. 1 and Table 4, both institutions scored highest on the NCC checklist in the infrastructure and available equipment categories, with hospital 1 highest in equipment (81.3\%) and hospital 2 highest in infrastructure $(69.6 \%)$. Both hospitals 1 and 2 scored lowest in the training/continuing education category $(41.7 \%$ and $33.3 \%$, respectively); the next lowest category for each hospital was processes (58.6\% and $34.5 \%$, respectively).

\section{Qualitative Assessment}

Qualitative assessment revealed capacity-development themes in 4 main categories: personnel, infrastructure, supplies, and equipment. Several themes specific to each category were identified.

\section{Personnel Themes}

Three personnel-related capacity-development themes emerged. The first theme identified was education. All interviewees suggested that educational initiatives for nurses and new physicians on topics pertaining to TBI care were needed. One provider remarked, "We have some trainings, but not enough. Sometimes help [is] needed for [the] nurse to know to call [the] doctor for brain injury patient." The top 4 commonly cited educational needs were conducting a bedside neurological examination, identifying signs that would warrant physician notification/intervention, ventilation of a patient with TBI, and management of intracranial devices such as external ventricular drains. The second personnel-related theme identified at both hospitals centered on the limited availability of experienced personnel to provide a high level of professional teaching, given conflicts with high case volumes and clinical obligations. The third personnel-related theme centered on staffing during the day versus evening shifts. Both physicians and nurses across all departments, with the exception of the emergency department, reported adequate staffing during the
TABLE 3. Summary of scores on the WHO PIPES assessment survey

\begin{tabular}{lccc}
\hline \multicolumn{1}{c}{ Category } & Hospital 1 & Hospital 2 & Total Possible Score \\
\hline Personnel & 44 & 32 & NA \\
\hline Infrastructure* $^{*}$ & 22 & 22 & 13 \\
\hline Procedure & 33 & 37 & 40 \\
\hline Supplies & 25 & 17 & 25 \\
\hline Equipment & 19 & 17 & 22 \\
\hline Total & 143 & 125 & NA \\
\hline Indext & 13.6 & 11.9 & NA \\
\hline
\end{tabular}

* There are 13 total infrastructure items; however, the number of operating rooms available is added to this score, which is why the hospital scores exceed the total possible score.

$\dagger$ Calculated by taking the total score, dividing by the number of survey items $(n=105)$, and multiplying by 10 : (total/105) $\times 10$.

day. However, interviewees frequently mentioned the importance of increasing evening staffing to similar ratios as during the day, given that evenings were particularly busy periods for both hospitals in general and TBI patients in particular.

\section{Infrastructure Themes}

Five different infrastructure themes emerged, some that were shared across subspecialties and others that showed a difference in emergency department versus ICU perspectives. First, all interviewees discussed providers' satisfaction with the breadth of subspecialty care offered at their respective institutions. This satisfaction was rooted in a culture of professionalism and perseverance and was characterized by camaraderie, collegiality, and creativity.

Second, participants identified inadequate unit size and bed capacity as significant barriers to providing highquality care in their facilities. In both the emergency and ICU departments of both hospitals, providers noted that the unit size was at times inadequate to meet the high patient volume, with both units commonly filled above capacity. At hospital 2 specifically, nurse-to-patient ratios were often reported as 1:6 given the high demand.

The third theme centered on prehospital admission care. Emergency department providers of both hospitals reported that many fatal injuries occurred in rural provinces that would otherwise have been survivable if more definitive TBI care was available during the long hospital transport. In their view, capacity-development initiatives focused on developing pre-hospital admission care of patients suffering from trauma, or initiatives that would lead to faster hospital presentation for definitive treatment, would lead to improvements in TBI outcomes; one interviewee said, "Sometimes the patient take too long to come; they maybe could live if they make it here fast, but there is trouble with this." These providers also desired protocols to organize and triage assessment upon patient arrival in the emergency department as important to TBI care capacity development.

The fourth and fifth themes involved establishment of isolation rooms and improvement of equipment maintenance systems. ICU personnel at both hospitals identified 


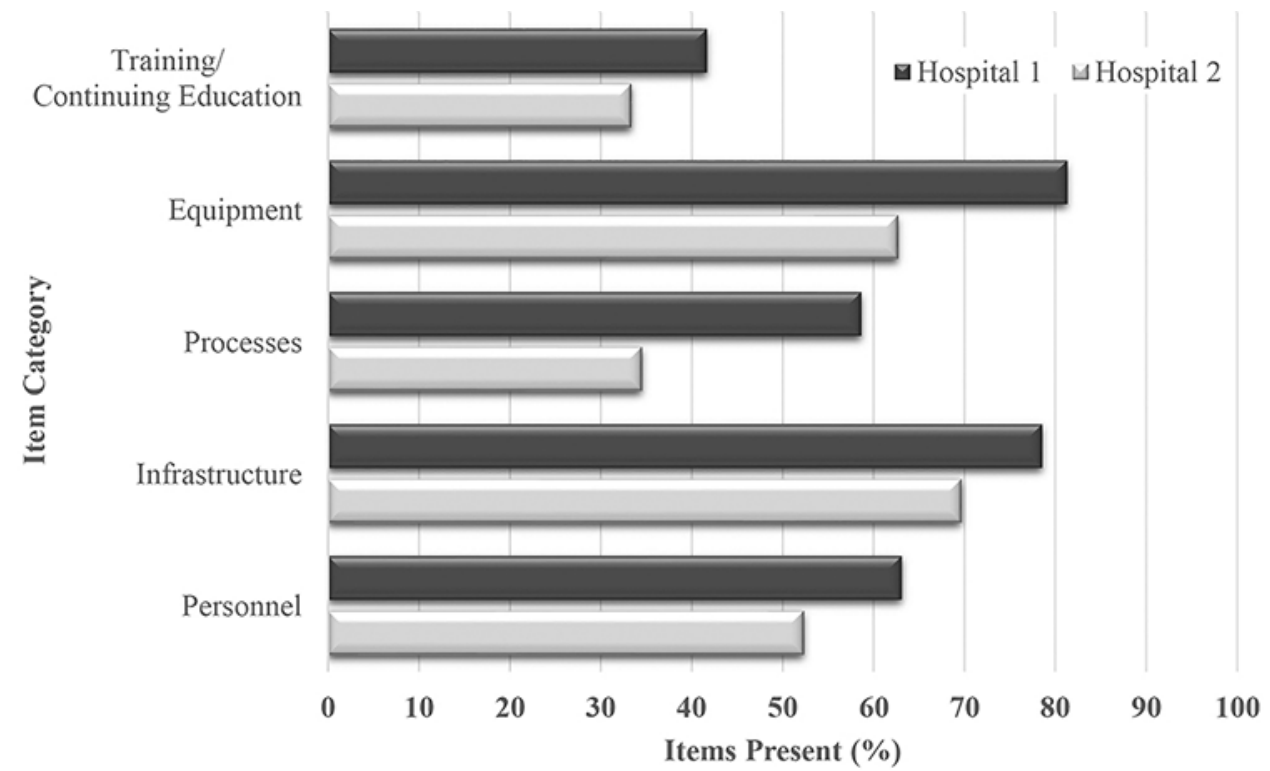

FIG. 1. Bar graph showing scores achieved on the NCC checklist by hospital 1 (black) and hospital 2 (light gray). Please see Table 4 for individual assessment numbers.

the development of standardized isolation rooms to enact infection protocols as a desired TBI capacity-development initiative. At hospital 2 specifically, ICU providers also frequently mentioned the need for improved equipment maintenance systems as important infrastructure developments that would contribute to improved TBI care. Interviewees from the neurology and neurosurgery departments of both hospitals echoed the infrastructure themes mentioned by both emergency and ICU providers.

\section{Supplies and Equipment Themes}

Supply and equipment themes varied between the two institutions. At hospital 1, although many supplies defined by the quantitative assessment tools were reportedly available, the rate-limiting factor expressed across all interviews in all departments was the patient's ability to pay for the materials and procedures required. For example, intracranial pressure monitors were reportedly available but so expensive that families could not afford the ICU stay, effectively rendering them obsolete. When asked if intracranial pressure monitors were available, one participant responded, "Yes, we had, and use all the time, but so expensive, no family could stay in ICU [afford the ICU stay]."

Equipment limitations were also nuanced; although providers reported having access to the equipment listed in the quantitative assessment, further discussion revealed providers commonly lacked the quantity required to allow simultaneous operations or to accommodate patients when a device was unavailable while being repaired. These limitations influenced operating room time; for example, a decompressive craniotomy was subjectively reported to take 40-60 minutes with an electric drill versus 3-4 hours with a Hudson brace and Gigli saw.

At hospital 2, supply and equipment limitations were a predominant theme in all provider interviews. While providers expressed appreciation for donations of disposable supplies and equipment, such as ventilators, defibrillators, and adjunctive respiratory tools from partnering institutions in HICs, they noted that there were inadequate working units to support the critical care demand; as one provider put it, "Sometimes no ventilator for our patient, so family have to [squeezes hand to signify bag masking]. Sometimes they fall asleep, tired, high $\left[\mathrm{CO}_{2}\right]$ is bad." Ad-

TABLE 4. Summary of NCC checklist items

\begin{tabular}{|c|c|c|c|c|c|c|c|}
\hline \multirow[b]{2}{*}{ Subdivision } & \multirow{2}{*}{$\begin{array}{l}\text { Total No. } \\
\text { of Items }\end{array}$} & \multicolumn{2}{|c|}{ No. of Items Present (\%) } & \multicolumn{2}{|c|}{ No. of Items Absent (\%) } & \multicolumn{2}{|c|}{ No. Unknown (\%) } \\
\hline & & Hospital 1 & Hospital 2 & Hospital 1 & Hospital 2 & Hospital 1 & Hospital 2 \\
\hline Personnel & 65 & $41(63.1)$ & $34(52.3)$ & $24(36.9)$ & $28(43.1)$ & 0 & $3(4.6)$ \\
\hline Infrastructure & 56 & $44(78.6)$ & $39(69.6)$ & $12(21.4)$ & $15(26.8)$ & 0 & $2(3.6)$ \\
\hline Processes & 29 & $17(58.6)$ & $10(34.5)$ & $12(41.4)$ & $18(62.1)$ & 0 & $1(3.4)$ \\
\hline Equipment & 75 & $61(81.3)$ & $47(62.7)$ & $14(18.7)$ & $25(33.3)$ & 0 & $3(4.0)$ \\
\hline Training/continuing education & 36 & $15(41.7)$ & $12(33.3)$ & $21(58.3)$ & $24(66.7)$ & 0 & 0 \\
\hline Total & 261 & 178 & 142 & 83 & 110 & 0 & 9 \\
\hline
\end{tabular}


ditionally, frequently these machines were older models of various designs with replacement parts that were sometimes discontinued or more expensive than the device itself, making equipment maintenance especially challenging.

\section{Discussion}

Combining quantitative and qualitative assessments in this mixed-methods study demonstrated that supplies, equipment, and education/training initiatives were the top 3 areas in which to focus capacity-development initiatives for neurosurgical care, and TBI care in particular, at two national hospitals in Phnom Penh, Cambodia.

\section{Benefits of a Mixed-Methods Study Design}

Although the quantitative assessments provided important insights, they did not fully capture the nuances or topics uncovered during the qualitative interview portion of the assessment, when native healthcare providers could directly voice areas they considered vital to capacity development. The mixed-methods study design thereby embodied the central tenet of the United Nations Capacity Development Primer that capacity development should focus on "empowering and strengthening endogenous capabilities"18 to ensure that initiatives and growth are generated from within the LMIC. For example, WHO PIPES and NeuroPIPES assessments suggested that improvements in the number of supplies, equipment, and generalization of provided services to include pediatric care would inform capacity-development initiatives for neurosurgical care. Qualitative assessment, however, demonstrated that issues of self-sustainability, maintenance, and affordability are critical for a lasting impact. The use of supplies and equipment was limited by the patient's ability to afford the service and by maintenance challenges due to model age and variety, similar to results reported by Perry and Malkin, in which $40 \%$ of equipment in developing countries was out of service for similar reasons. ${ }^{15}$ Additionally, the importance of educational initiatives expressed during qualitative interviews would have been missed entirely if only PIPES assessments were utilized.

\section{Applicability of the NCC Checklist}

This study demonstrates the first application of the NCC checklist in an LMIC setting, and it provides insight into the applicability of definitions of a Level 1 NCCU in this setting. The NCS recognizes 3 levels of NCCU, with Level 1 units defined as "receiving centers for patients with complex neurological emergencies who require advanced interventions and provide the most comprehensive neurocritical care." 10 NCS requires Level 1 units to fulfill all checklist criteria except for 6 items that are optional: under the equipment category, brain tissue oxygen monitoring, cerebral blood flow monitoring, and microdialysis; under the training/research category, having a neurology residency and both a fellowship program and clinical rotation in neurocritical care. By one definition, both hospitals in this study qualify as Level 1 units, since they are tertiary referral centers for complex cases requiring subspecialty care. However, based on the results of the NCC checklist, these hospitals lacked access to requirements that include EEG machines, helipads, neurointerventionalists, and NCCU pharmacists. Adjustment of these requirements in the NCC checklist to reflect resource limitations experienced by LMICs would allow this classification scheme to remain relevant in these settings. As nations gain wealth, scaling requirements for a Level 1 NCCU based on a country's World Bank classification might provide a useful guide for capacity-development goals.

Since the NCC checklist does not significantly overlap with the WHO PIPES and NeuroPIPES tools, its coadministration provided important additional information to assess the nonoperative and perioperative care of neurosurgical patients. ${ }^{10,16}$ Furthermore, the NCC checklist demonstrated that training and education of staff reflected an important capacity-development initiative in this setting. It could be argued that this result is explained by specific certifications and schooling requirements listed on the NCC checklist and available in HIC institutions but that were otherwise unavailable in LMICs, such as the Emergency Neurological Life Support certification. However, qualitative interview results demonstrated that native providers also noted a dearth of staff educational programs focused on neurological pathologies, and providers considered improvement in this category important to improving care for patients with TBI. "Twinning" an HIC with an LMIC to build technology capacity and provide training and education has been reported as a useful strategy for building neurosurgical capacity in an LMIC. ${ }^{7}$ Further research on this approach is warranted.

\section{Site-Specific Considerations}

Despite similarities, each hospital was shown to have unique barriers to capacity development. For example, providers in hospital 1 mentioned the financial barrier of patients' inability to afford expensive procedures, whereas hospital 2 providers emphasized the material barrier of requiring more supplies and equipment. This, combined with the difference in the NCC checklist "processes" category comparing hospital 2 with hospital 1, suggests that different prioritizations of capacity-development initiatives for each institution may be appropriate. Further evaluation will be necessary.

\section{Limitations}

This study has some limitations. The interviews were administered in English, a second or third language for all participants. The language barrier may have limited the number of available participants, thereby introducing a potential selection bias. Language differences may have prevented elucidation of nuances in perspectives among participants; however, efforts were made to ensure a saturation of responses across themes and participants. Further research is also needed to validate comparisons between each assessment tool utilized.

\section{Conclusions}

In these two Cambodian hospitals, this study identified several opportunities for capacity development in the care of the TBI population within the assessed categories 
of supplies, equipment, and training/educational initiatives. This study also demonstrated that barriers unique to each institution may influence prioritization of implementing capacity-development projects. The inclusion of the NCC checklist was particularly useful in identifying educational initiatives as an area in which to focus capacity development; however, its use points out limitations in current NCCU classifications in the setting of LMICs. Although time intensive, the mixed-methods study design allowed the combination of quantitative and qualitative data to provide important insights into barriers verbalized by native healthcare professionals that would otherwise have remained undetected using a single methodology.

\section{Acknowledgments}

We appreciate the assistance of Sharon Durfy, $\mathrm{PhD}$, with manuscript preparation.

\section{References}

1. Albert TJ, Fassier T, Chhuoy M, Bounchan Y, Tan S, Ku N, et al: Bolstering medical education to enhance critical care capacity in Cambodia. Ann Am Thorac Soc 12:491-497, 2015

2. Braun V, Clarke V: Teaching thematic analysis: overcoming challenges and developing strategies for effective learning. Psychologist 26:120-123, 2013

3. Braun V, Clarke V: Using thematic analysis in psychology. Qual Res Psychol 3:77-101, 2006

4. Carney N, Totten AM, O'Reilly C, Ullman JS, Hawryluk GW, Bell MJ, et al: Guidelines for the Management of Severe Traumatic Brain Injury, Fourth Edition. Neurosurgery 80:6-15, 2017

5. Dewan MC, Rattani A, Gupta S, Baticulon RE, Hung YC, Punchak M, et al: Estimating the global incidence of traumatic brain injury. J Neurosurg 130:1080-1097, 2019

6. Groen RS, Kamara TB, Dixon-Cole R, Kwon S, Kingham TP, Kushner AL: A tool and index to assess surgical capacity in low income countries: an initial implementation in Sierra Leone. World J Surg 36:1970-1977, 2012

7. Haglund MM, Kiryabwire J, Parker S, Zomorodi A, MacLeod D, Schroeder R, et al: Surgical capacity building in Uganda through twinning, technology, and training camps. World J Surg 35:1175-1182, 2011

8. Maguire M, Delahunt B: Doing a thematic analysis: a practical, step-by-step guide for learning and teaching scholars. All Ireland J Higher Educ 9:3351-33514, 2017 (http://ojs.aishe.org/index.php/aishe-j/article/view/335/553) [Accessed October 25, 2019]

9. Meara JG, Leather AJM, Hagander L, Alkire BC, Alonso N, Ameh EA, et al: Global Surgery 2030: evidence and solutions for achieving health, welfare, and economic development. Lancet 386:569-624, 2015

10. Moheet AM, Livesay SL, Abdelhak T, Bleck TP, Human T, Karanjia N, et al: Standards for neurologic critical care units: a statement for healthcare professionals from the Neurocritical Care Society. Neurocrit Care 29:145-160, 2018

11. Norton R, Kobusingye O: Injuries. N Engl J Med 368:17231730, 2013

12. Park KB, Johnson WD, Dempsey RJ: Global neurosurgery: the unmet need. World Neurosurg 88:32-35, 2016

13. Patel HC, Bouamra O, Woodford M, King AT, Yates DW, Lecky FE: Trends in head injury outcome from 1989 to 2003 and the effect of neurosurgical care: an observational study. Lancet 366:1538-1544, 2005
14. Peeters S, Blaine C, Vycheth I, Nang S, Vuthy D, Park KB: Epidemiology of traumatic brain injuries at a major government hospital in Cambodia. World Neurosurg 97:580-589, 2017

15. Perry L, Malkin R: Effectiveness of medical equipment donations to improve health systems: how much medical equipment is broken in the developing world? Med Biol Eng Comput 49:719-722, 2011

16. Ploss B, Abdelgadir J, Smith ER, Fuller A, Nickenig Vissoci JR, Muhindo A, et al: Pilot use of a novel tool to assess neurosurgical capacity in Uganda. World Neurosurg 108:844849.e4, 2017

17. Rubiano AM, Carney N, Chesnut R, Puyana JC: Global neurotrauma research challenges and opportunities. Nature 527:S193-S197, 2015

18. United Nations Development Programme: Capacity Development: A UNDP Primer. 2009 (https://www.undp. org/content/dam/aplaws/publication/en/publications/capacitydevelopment/capacity-development-a-undp-primer/CDG_ PrimerReport_final_web.pdf) [Accessed October 25, 2019]

19. World Bank: Cambodia GNI (current US\$). 2017 (https:// data.worldbank.org/indicator/ny.gnp.mktp.cd) [Accessed October 25, 2019]

20. World Health Organization: Global Status Report on Road Safety 2013: Supporting a Decade of Action. 2013 (https:// www.who.int/violence_injury_prevention/road_safety_ status/2013/en/) [Accessed October 25, 2019]

21. World Health Rankings: Cambodia: life expectancy. 2018 (https://www.worldlifeexpectancy.com/cambodia-lifeexpectancy) [Accessed October 25, 2019]

\section{Disclosures}

Dr. Spece reports receipt of funding from the National Heart, Lung, and Blood Institute (grant no. 5K12HL137940). Dr. Lele reports receipt of funding from Aqueduct Critical Care and salary support from LifeCenter Northwest.

\section{Author Contributions}

Conception and design: Barkley, Spece, Barros, Bonow. Acquisition of data: Barkley, Huoy, Thy, Sothea, Pak. Analysis and interpretation of data: Barkley. Drafting the article: Barkley. Critically revising the article: all authors. Reviewed submitted version of manuscript: all authors. Approved the final version of the manuscript on behalf of all authors: Barkley. Study supervision: LoGerfo, Lele.

\section{Supplemental Information \\ Online-Only Content}

Supplemental material is available with the online version of the article.

Supplementary Table 1. https://thejns.org/doi/suppl/10.3171/ 2019.10.JNS192118.

\section{Correspondence}

Ariana S. Barkley: University of Washington, Seattle, WA. respub@uw.edu. 